\title{
Long-term rearrangement of retinal structures in a novel mutation of $\mathrm{X}$-linked retinoschisis
}

\author{
STEFANO PIERMAROCCHI $^{1}$, STEFANIA MIOTTO $^{2}$, DAVIDE COLAVITO $^{3}$, ELDA DEL GIUDICE $^{3}$, \\ ALBERTA LEON $^{3}$, VERONICA MARITAN $^{4}$, RITA PIERMAROCCHI $^{5}$ and ALMA PATRIZIA TORMENE ${ }^{1}$ \\ ${ }^{1}$ University Eye Clinic, University of Padua, I-35128 Padua; ${ }^{2}$ Ophthalmic Department, Unità Locale \\ Socio-Sanitaria 6 Euganea, Camposampiero, I-35012 Padua; ${ }^{3}$ Research and Innovation \\ (R\&I Genetics) Srl, I-35127 Padua; ${ }^{4}$ Ophthalmic Department, ULSS 6 Euganea, Monselice, \\ I-35043 Padua; ${ }^{5}$ Ophthalmic Department, ULSS 5 Polesana, I-45100 Rovigo, Italy
}

Received May 30, 2017; Accepted June 6, 2017

DOI: $10.3892 /$ br.2017.954

\begin{abstract}
The aim of the present study was to report a novel mutation in the retinoschisin 1 (RS1) gene in a Caucasian family affected by X-linked juvenile retinoschisis (XLRS) and to describe the long-term modification of retinal structure. Two brothers with an early onset maculopathy were diagnosed with XLRS. Fundus photography, fluorescein angiography, spectral domain optical coherence tomography and electroretinogram analyses were performed. Their sister was also examined. All subjects were screened for mutations in the RS1 gene. XLRS patients demonstrated a marked reduction of best-corrected visual acuity. SD-OCT scans reported a cystic degeneration primarily involving the inner nuclear layer, though some cysts were detected in the outer plexiform layer and in the ganglion cell layer. During the ten-year follow-up, a progressive retinal thickening and coalescence of the cysts was observed. Genetic testing revealed a novel mutation (p.Ile212Asn) in the RS1 gene in both XLRS patients, whereas their sister was not a genetic carrier. Several mutations of the RS1 gene were recognized to be responsible for XLRS. Although the correspondence between genotype and phenotype is still under debate, is reasonable that siblings affected by XLRS could share other genetic and/or epigenetic factors capable to influence clinical course of the disease.
\end{abstract}

\section{Introduction}

X-linked juvenile retinoschisis (XLRS, phenotype MIM 312700), first described in 1898 by Joseph Haas, represents the leading cause of early macular degeneration in males $(1,2)$. Its prevalence ranges from 1:5,000 to 1:25,000 (3).

Correspondence to: Dr Davide Colavito, Research and Innovation (R\&I Genetics) Srl, Corso Stati Uniti 4, I-35127 Padua, Italy

E-mail: colavito@researchinnovation.com

Key words: X-linked retinoschisis, RS1 gene, retinoschisin, inner nuclear layer
Bilateral splitting of the inner retina is the most common clinical finding and has been reported in 68-100\% of affected males. In ophthalmoscopy, the macula presents stellate spokewheel pattern. Female carriers rarely present with retinal abnormalities (4). As the disease progresses, retinal cysts often coalesce with subsequent retinal flattening and macular atrophy in older patients. Peripheral schisis, usually located in the inferotemporal retina, is evident in $\sim 50 \%$ of the affected individuals (5). Although this condition is considered to be congenital, symptoms generally present in the first decade of life with visual failure, squint or nystagmus. Visual damage usually progresses in the first two decades of life and remains approximately stationary until the fifth or sixth decade, when the development of macular atrophy induces additional visual loss (3).

Patients with XLRS present a characteristic pattern on the electroretinogram (ERG). A reduction in the amplitude of the b-wave (generated by the activity of depolarising bipolar cells) and a relative preservation of the negative a-wave (generated by photoreceptors) give rise to, the so-called electronegative ERG (6). The absence of negative ERG does not necessarily exclude the presence of the pathology: in a series of 24 XLRS patients, only $56.5 \%$ presented with typical negative ERG (7).

Optical coherence tomography (OCT) is a key diagnostic test for XLRS. Schisis seems to occur predominantly at the inner nuclear layer (INL), occasionally at the outer nuclear layer/outer plexiform layer (ONL/OPL), and only rarely at the retinal nerve fiber layer (RNFL). The schisis cavity may extend beyond the retinal vascular arcades (8).

The gene responsible for XLRS, retinoschisin 1 (RS1), was identified by Sauer et al (2) in 1997. The RS1 gene, on chromosome Xp22, encodes for retinoschisin, a discoidindomain containing protein, which is secreted by photoreceptors and bipolar cells as a homo-oligomeric complex $(2,9,10)$. Retinoschisin complex binds tightly to the surface of photoreceptors and bipolar cells, contributing to maintain the structural organization of the retina and of the photoreceptorbipolar synapse (4). In people with XLRS, several missense and nonsense mutations, insertion and deletion mutations, intragenic deletions and splice site mutations have been identified (11). 
The aim of the present study is to describe clinical features of a family affected by the novel missense mutation I $212 \mathrm{~N}$ of the RS gene and to provide a detailed follow-up based on spectral domain optical coherence tomography (SD-OCT).

\section{Materials and methods}

Patients. The present study included a Caucasian Italian family with two brothers clinically affected by XLRS. Both patients underwent a complete ophthalmological assessment and were followed up for ten years, including best-corrected visual acuity (BCVA) and fundus examination. Detailed multimodal retinal imaging (fundus photography, OCT, fundus autofluorescence (FAF) and retinal angiography) was collected. Moreover, retinal function was evaluated with ERG and Goldmann perimetry. Clinical and genetic testing was extended to other family members. The current study was conducted in accordance with The Declaration of Helsinki.

XLRS1 gene analysis. Written informed consent for genetic analysis was obtained from the all subjects. Whole blood samples were then collected. DNA was extracted using the Qiagen Biorobot DNA extraction kit (Qiagen, Inc., Valencia, CA, USA) according to manufacturer's instructions and quantified by Nanodrop spectral analysis (Thermo Fisher Scientific, Inc., Waltham, MA, USA). DNA fragmentation and degradation were evaluated by standard agarose gel electrophoresis. A total of $100 \mathrm{ng}$ DNA were amplified by standard polymerase chain reaction (PCR) procedures with a PCR mixture containing 2.5 $\mu 110 \mathrm{X}$ concentrated PCR buffer (Solis BioDyne,

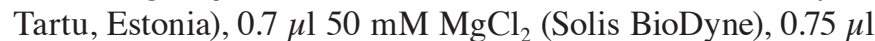
$10 \mathrm{mM}$ deoxyribonucleotide triphosphates (Solis BioDyne), $0.3 \mu \mathrm{l} 100 \mu \mathrm{M}$ forward primer and $0.3 \mu \mathrm{l}$ of $100 \mu \mathrm{M}$ reverse primer (primer sequences are listed in Table I) (Integrated DNA Technologies, Coralville, IA, USA) and $0.5 \mu 15 \mathrm{U} / \mu 1$ Hot Start DNA Polymerase (Solis BioDyne). Thermocycling consisted of one cycle of enzyme activation (at $95^{\circ} \mathrm{C}$ for $15 \mathrm{~min}$ ), followed by 35 cycles of DNA amplification (at $95^{\circ} \mathrm{C}$ for $45 \mathrm{sec}$, at $59^{\circ} \mathrm{C}$ for $45 \mathrm{sec}$ and at $72^{\circ} \mathrm{C}$ for $1 \mathrm{~min}$ ). PCR products were then separated by agarose gel electrophoresis (1.5\% agarose gel in tris-borate-EDTA; Sigma-Aldrich; Merck KGaA, Darmstadt, Germany) and purified with Invisorb spin columns (Invitek, Inc., Hayward, CA, USA). PCR-purified products were re-amplified with terminating nucleotides using Big Dye Terminator v3.1 (Applied Biosystems; Thermo Fisher Scientific, Inc.). Sequencing analysis was performed with an ABI Prism 3100 Avant automated sequencer (Thermo Fisher Scientific, Inc.) equipped with $36 \mathrm{~cm}$ capillary array filled with POP6 polymer (Thermo Fisher Scientific, Inc.). Electropherograms were analyzed using Sequencing Analysis software (Applied Biosystems, version 5.1; Thermo Fisher Scientific, Inc.).

\section{Results}

Patients. Three members of a family with two brothers affected by XLRS were studied. The first proband was a 41-year-old male and complained of mild visual disturbance since the age of 9, where medical records reported a spoke-wheel appearance of the macula and a BCVA of 0.8 . His medical history was
Table I. Primer sequences.

\begin{tabular}{ll}
\hline Primer & \multicolumn{1}{c}{ Sequence (5'-3') } \\
\hline RS1_exon 1F & GGAAAGCCATCCACACAAAG \\
RS1_exon 1R & GGTTAACTTGATGGGGCTCA \\
RS1_exon 2F & TCCTGACCTCAAGTGATCTGC \\
RS1_exon 2R & TTCTTCCAGAAGGGGTGTTG \\
RS1_exon 3F & GGAGAAAACCCGCATTAACA \\
RS1_exon 3R & GACGATGCATAAGGACTGAGTG \\
RS1_exon 4F & CCACCACGCCAGTTAATTTT \\
RS1_exon 4R & GCAAAGCAGATGGGTTTGTT \\
RS1_exon 5F & ACAGAGGGCAGTGACAGGAG \\
RS1_exon 5R & GGAGACAAGGCTCAGACTGC \\
RS1_exon 6F & ACCCAGCACTGCAGTTACAA \\
RS1_exon 6R & GGGCTAGCTCCAGAAAGGAA
\end{tabular}

F, forward; R, reverse.

unremarkable. At the first presentation, BCVA was 0.4 in oculus uterque and remained stable for all the follow-up (2007 through to 2016). Fundus examination reported the typical spoke wheel appearance of the macula in both eyes. In the right eye, a peripheral schisis in the inferior-temporal retina reaching the vascular arcade was also observed. Macular abnormalities were best recognized with FAF that indicated hyper- and hypo-FAF arranged in a radial pattern. Fluorescein angiography did not reveal any late leakage and confirmed the non-exudative origin of macular cysts.

On first examination with OCT, macular thickness was significantly increased. OCT scans presented a cystic degeneration, primarily involving the INL, though some small cysts were detected in the outer plexiform layer (OPL) and in the ganglion cell layer (GCL). In RE, peripheral scans documented retinal schisis extending inferiorly. The first OCT was acquired when the patient was $\sim 31$ years old and BCVA was already reduced. In the right eye, the ten-year follow-up demonstrated that there were no noticeable variations in retinal morphology or in the overall width of the peripheral schisis. However, the progressive straining of retinal layers breaks up the wall of macular cysts, weakening the retinal structure. Indeed, in the left eye, there was a moderate reduction in central retinal thickness (from $448 \mu \mathrm{m}$ to $402 \mu \mathrm{m}$ ) that precedes the late, atrophic stage of XLRS. Outer retinal layers (external limiting membrane, inner segment/outer segment junction and photoreceptor layer) demonstrated diffuse atrophic changes with no significant progression during the follow-up (Fig. 1). Goldmann perimetry indicated in RE an absolute scotoma superiorly, that matched the peripheral retinal schisis. The dark-adapted 0.01 ERG (rod response) was reduced. The dark-adapted 3.0 ERG (combined rod-cone response) showed a b/a ratio $<1$, which matched the definition of an electronegative ERG. The patient reported a delay in implicit time and a decrease in the amplitude of the b-wave in the light adapted 3.0 ERG. A delayed light adapted $30 \mathrm{~Hz}$ flicker peak time and decreased amplitude was also present. The diagnosis of XLRS was proposed and all family members were then invited for eye examination. His brother was a 30-year-old male. The diagnosis of unspecified retinal 

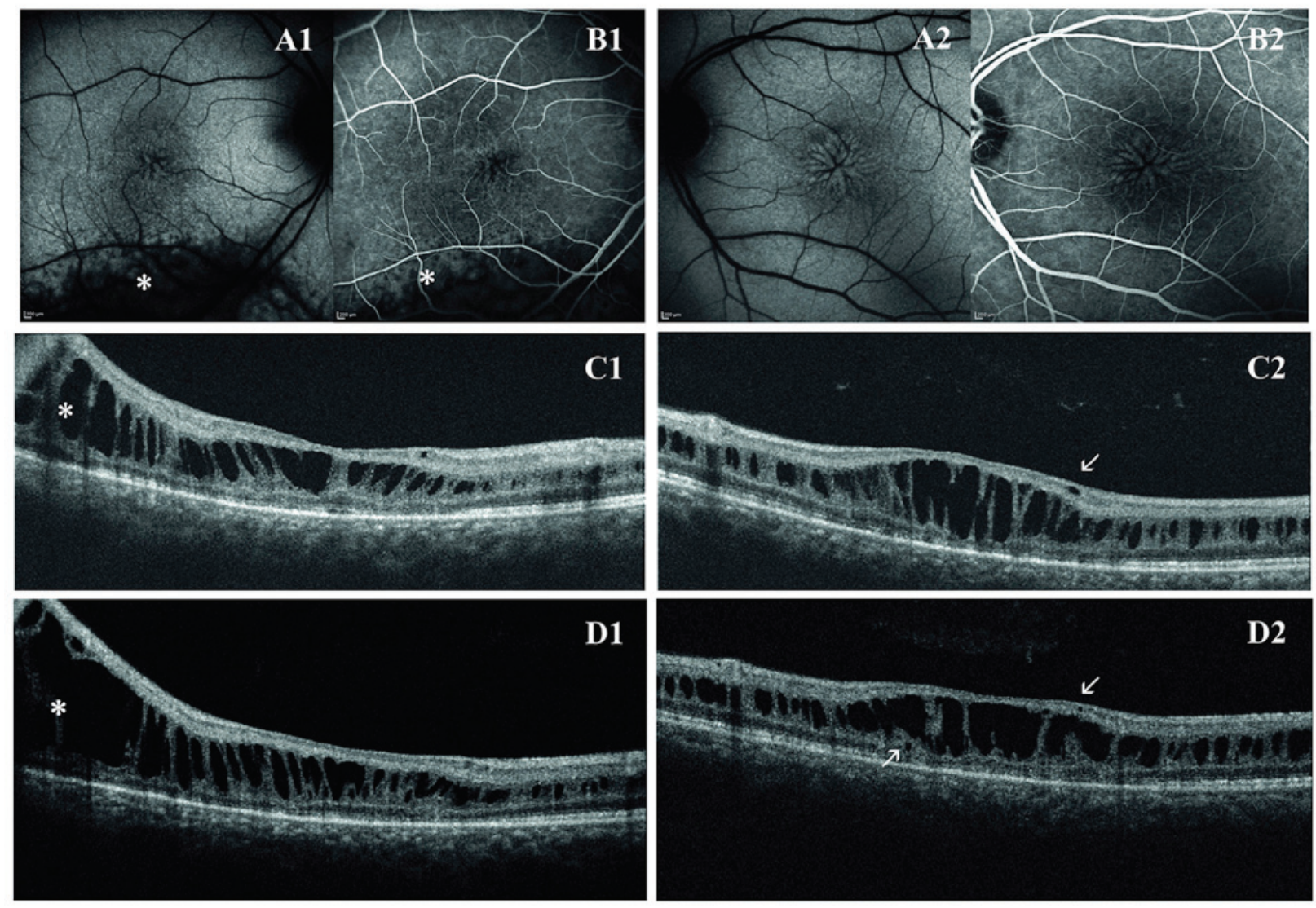

Figure 1. First proband. Spoke wheel appearance of the macula as seen using (A1, A2) fundus autofluorescence and (B1, B2) fluorescein angiography (transverse resolution, $14 \mu \mathrm{m}$ ). In the right eye is a recognizable peripheral schisis in the inferior-temporal retina as indicated by *). Optical coherence tomography scans at the first examination reported a cystic degeneration primarily involving the inner nuclear layer in the (C1) right eye and in the (C2) left eye. Some small cysts are visible in the ganglion cell layer and outer plexiform layer (arrows). In the ten-year follow-up, the (D1) right eye did not show significant variations of retinal thickness and of peripheral schisis width, whereas in the (D2) left eye, there is a little reduction in central retinal thickness, due to coalescence of retinal cysts and initial collapse of retinal structure (axial resolution, $6 \mu \mathrm{m}$ ).

dystrophy was made when he was 8 years old and he presented with a initial visual loss associated with characteristic spoke wheel appearance of the macula. At the examination, BCVA was 0.5 in RE and 0.6 in LE and progressively decreased to 0.4 in RE and 0.3 in LE during follow-up. Fundus examination, OCT and retinal angiography revealed the typical features of XLRS. An electronegative ERG further supported the diagnosis. First OCT scans presented irregular empty spaces in the INL. Some small cysts could be observed in the GCL. Atrophic changes involving outer retinal layers (external limiting membrane, inner segment/outer segment junction and photoreceptor layer) were recognizable. During the followup, the OCT scans revealed a progressive enlargement and coalescence of the cysts located in the INL, with subsequent retinal thickening. Small cysts in the GCL did not show further enlargement. Atrophic changes of outer retinal layers remained stable during follow-up (Fig. 2).

Ophthalmologic examination of their sister (21 years old) and of their father (69 years old) was normal. Their mother was deceased, but no significant visual disturbances were reported, even though it was not possible to ascertain if she was a healthy carrier or if she developed a de novo mutation. Indeed, ocular diseases in their maternal grandfather were not reported. Then, the authors drew a genealogical tree based on available information (Fig. 3).
Genetic testing. Screening of RS1 by sequencing of PCR-amplified DNA identified a novel missense mutation in exon 6 in both XLRS patients. This mutation from A to $\mathrm{T}$ at nucleotide position 635 changes the nonpolar isoleucine to positively charged asparagine (p.Ile212Asn).

This substitution was predicted to be harmful to protein function by in silico analysis (Polyphen2=0.989; Fig. 3).

\section{Discussion}

Mutations in the RS1 gene are responsible for inherited and sporadic XLRS. To date 191 causative genetic variations have been identified. Among all described variations, missense mutations represent the most recurrent subtype (100 mutations of 191). Mutations can affect all regions of the RS gene, although a substantial clustering is observed within the region coding for the discoidin domain (85 of 191) (4). The novel missense mutation that we found in the patients is capable to produce the typical clinical phenotype of XLRS, as it affects the discoidin domain of retinoschisin.

Retinoschisin is a 24-kDa protein expressed exclusively by photoreceptors and bipolar cells in the retina and pineal gland. RS1 is secreted as a soluble disulfide bond-stabilized octamer. Most of the monomer (157 amino acids) comprises a discoidin domain, a globular fold that is highly conserved in a 

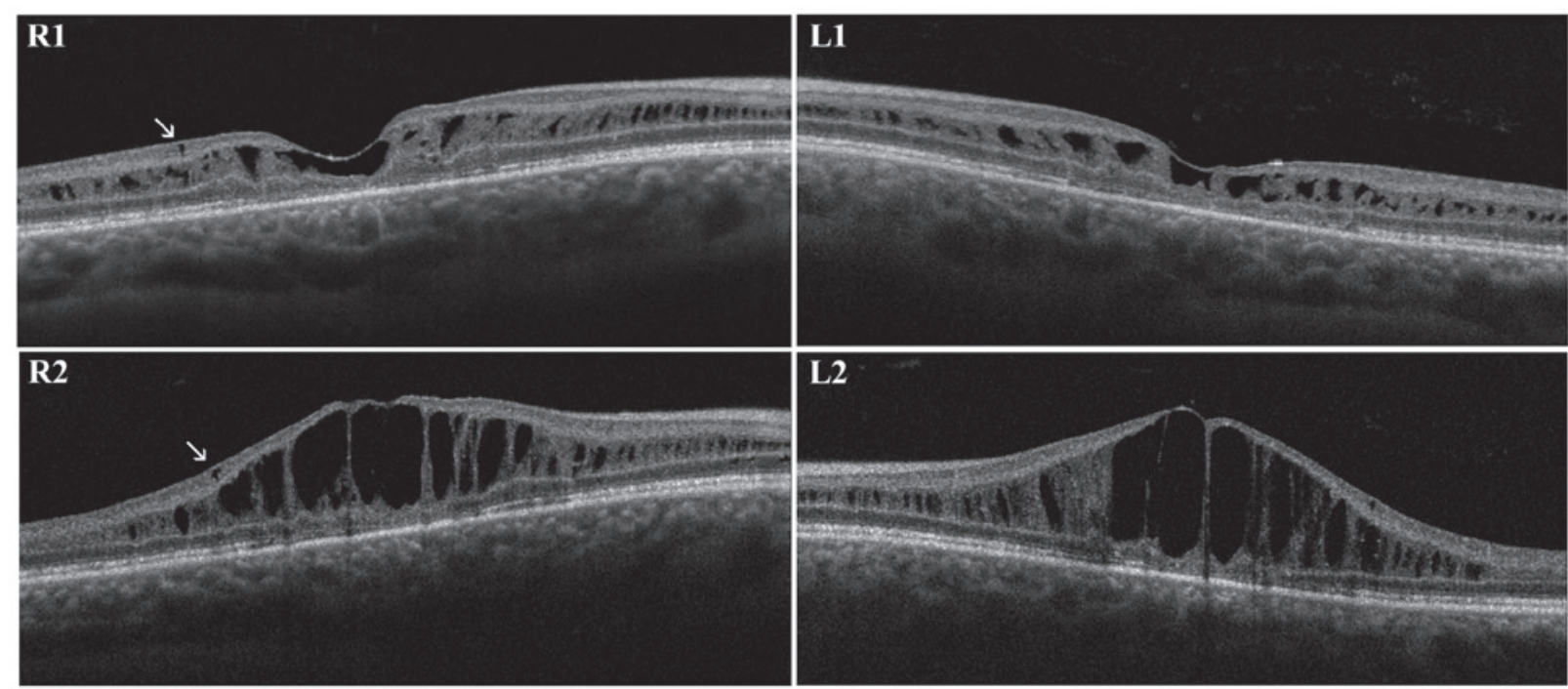

Figure 2. Second proband. First optical coherence tomography scans in the (R1) right eye and (L1) left eye reported irregular cysts in the INL and some small cysts in the GCL (arrows). The ten-year follow-up displayed a progressive enlargement and coalescence of the cysts located in the INL with subsequent retinal thickening. Small cysts in the GCL remained unchanged (arrows). INL, inner nuclear layer; GCL, ganglion cell layer (axial resolution, $6 \mu \mathrm{m}$ ).

family of extracellular or transmembrane proteins implicated in cell adhesion or cell-cell interactions. The high number of mutations within the discoidin domain indicates that it is essential for the normal function of this protein. Although the role of retinoschisin in the retina is not well understood, it has been hypothesized that it works as a cell adhesion protein to maintain the structural organization of the retina and of the photoreceptor-bipolar synapse $(4,5,10,12)$.

Differential diagnosis of XLRS comprises cystic changes of the macula that could arise from exudative and degenerative disorders or abnormalities of the vitreoretinal interface. Degenerative cystoid maculopathies may be hereditary [XLRS, Enhanced S-Cone/Goldmann Favre Syndrome (ESCS/GFS)] or acquired [microcystic macular edema (MME)]. All these conditions do not show late leakage on fluorescein angiography.

ESCS/GFS is a recessive disorder caused by mutations in the NR2E3 gene. This gene encodes for a nuclear receptor expressed in the outer nuclear layer of the retina. It suppresses cone differentiation during embryogenesis, therefore loss of NR2E3 results in retinas with a decreased number of rod photoreceptors and an increase in cones, predominantly expressing the S-cone opsin (13). Time trend of macular cysts development in ESCS/GFS resemble what happens in XLRS. The progressive enlargement and coalescence of macular cysts in young adulthood is followed by resolution of the schisis and reduction of macular thickness (14). However, location of cystic spaces seems different from XLRS. The analysis of published images shows large confluent cysts in the outer retinal layer associated with small and well-demarcated cysts in the INL $(15,16)$.

MME is a recently described OCT entity, characterized by the appearance of small retinal cysts in patients with optic neuritis and optic atrophy of various aetiology (multiple sclerosis, neuromielitis optica, glaucoma) probably due to retrograde synaptic degeneration. As in XLRS, MME cysts involve the INL, however they do not seem to enlarge significantly with time. Moreover in MME cysts are usually circumscribed to the parafoveal region $(17,18)$, whereas in XLRS cystic degeneration may extend beyond the vascular arcades.

Diagnosis of XLRS in the patients of the present study was strongly supported by clinical and instrumental findings. Indeed both patients presented with the classical phenotype of XLRS, with spoke-wheel maculopathy, association with peripheral schisis and ERG b-wave suppression. Ophthalmological history, regarding age of onset of symptoms and visual decline follows the typical course described in literature (7). Visual symptoms begin in the first decade, and then visual function progressively decreases, remaining stable in the following 10 years.

SD-OCT images collected in the two patients documented the natural history of the disease, characterized by the formation of small cysts located in the INL that progressively enlarge and coalesce. Gradually, the stretching of the wall of macular cysts weakens the retinal structure leading to the collapse of the cysts. Small isolated cysts were also observed in the GCL and OPL that, differently from those located in the INL, do not seem to increase over time. The possible presence of small cysts not determining macular splitting have been previously described by Gregori et al (19), who also found similar empty spaces in the ONL and in the GCL. The same paper reported that macular schisis may occur also in the OPL (19). An immunochemistry study (20) in the normal mouse eye detected retinoschisin protein in all retinal layers; this may explain why retinal schisis can develop in different layers, even if some layers (GCL and RNFL) seem relatively resistant. The foveomacular retinoschisis is usually located in the INL, while the extramacular schisis could be equally found in the INL, ONL and GCL/NFL (21). In the present study, cystic degeneration at the level of peripheral schisis in RE of the first proband was located in the INL (Fig. 1).

Since clinical and instrumental findings were highly suggestive for XLRS, the authors performed genetic analysis. The sequencing of the proband DNA, revealed the hemizygous 

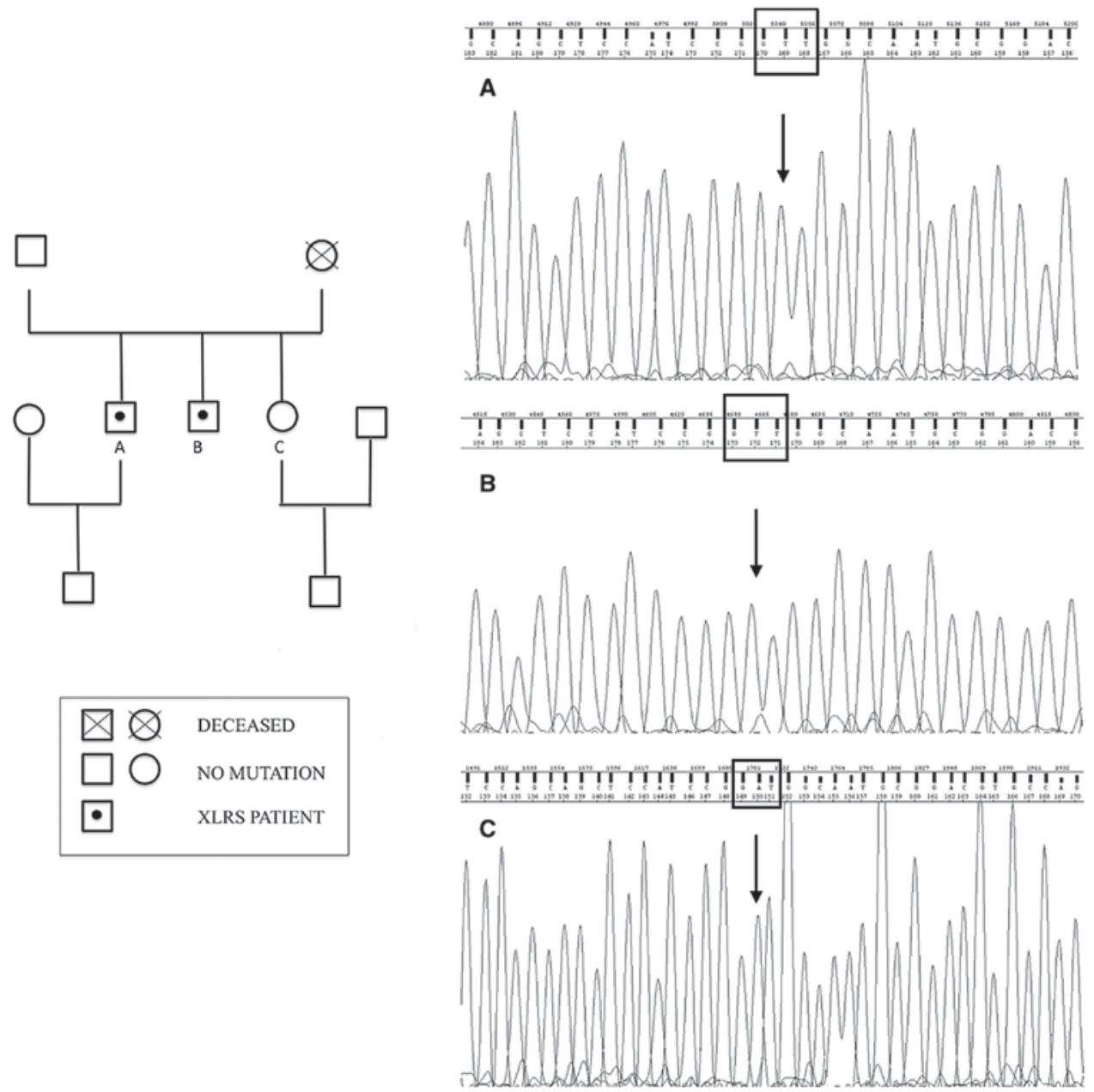

Figure 3. Family pedigree of a Caucasian family with (left) XLRS (left) and (right) p.Ile212Asn electropherograms. The first and second proband are named $\mathrm{A}$ and $\mathrm{B}$, respectively, whereas their sister is named C. XLRS, X-linked juvenile retinoschisis.

missense mutation p.1le212Asn (c.635A $>$ T) in the RS1 gene. The same mutation was identified in the proband's brother and has never been reported before. It consists in a substitution of the non-polar amino acid, isoleucine, with the polar amino acid, asparagine. The mutation is located in a domain in which several genetic alterations associated with XLRS has been found (positions p.206, p.209, p.211, p.213, p.2015 and p.219). Furthermore, the PolyPhen-2 predictor has given to this mutation a high likelihood of protein dysfunction.

Given the high number of RS1 mutations, several studies investigated a possible correlation between genotype and phenotype. Even though some reports describe a correlation between specific variations and the severity of clinical phenotype $(22,23)$, the majority of published studies found that XLRS patients had relatively uniform clinical manifestations, although with great intra-familial variation in age at onset and progression (1,24-26).

Interestingly, the deletion of exon 1 and promoter region, which causes complete absence of retinoschisin, is associated with a wide variability of the phenotype, suggesting that other genetic and/or epigenetic factors are likely to act as significant phenotypic modifiers in XLRS $(27,28)$.

The absence of leakage on fluorescein angiography in XLRS patients suggests that vascular hyperpermeability serves a minor role, if any, in the pathogenesis of the disease. However, both oral and topical carbonic anhydrase inhibitors (CAIs) have been used successfully in the management of XLRS. CAIs act both on retinal and RPE cell function by acidifying the subretinal space, decreasing the standing potential as well as raising retinal adhesiveness, probably by increasing RPE fluid transport (29).

A promising approach for XLRS treatment is gene therapy. The disease is an excellent candidate for gene therapy as the majority of mutations have been shown to lead to a complete deficiency of the secreted protein in the retinal structures. Genetic treatment studies, conducted in rabbits and mouse, with intravitreal administration of adeno-associated viral vector coding the human RS1 gene demonstrated an improvement in retinal structure and function $(30,31)$. Other strategies comprise the intravitreal administration of adiposederived mesenchymal stem cells, genetically modified to secrete the human RS1 gene, or solid lipid nanoparticles inducing the expression of retinoschisin in photoreceptors. Both treatments demonstrated morphological and functional improvements in mouse models $(32,33)$. Two phase I/II trials are testing safety and efficacy of adeno-associated viral vectors, called AAV8-scRS/IRBPhRS and rAAV2tYF-CB-hRS1, as a gene therapy approach in XLRS patients. Viral vectors have 
been demonstrated to be able to shuttle normal RS1 DNA into retinal cells. Results are expected from 2017 (34,35).

The present study describes long-term morphological and functional changes of XLRS patients affected by a novel RS1 mutation. Although the correspondence between genotype and phenotype is still under debate, is reasonable that siblings affected by XLRS could share other genetic and/or epigenetic factors capable to influence clinical course of the disease and perhaps treatment response to upcoming genetic therapies.

\section{References}

1. The Retinoschisis Consortium: Functional implications of the spectrum of mutations found in 234 cases with X-linked juvenile retinoschisis. Hum Mol Genet 7: 1185-1192, 1998.

2. Sauer CG, Gehrig A, Warneke-Wittstock R, Marquardt A, Ewing CC, Gibson A, Lorenz B, Jurklies B and Weber BH: Positional cloning of the gene associated with X-linked juvenile retinoschisis. Nat Genet 17: 164-170, 1997.

3. Mooy CM, Van Den Born LI, Baarsma S, Paridaens DA, Kraaijenbrink T, Bergen A and Weber BH: Hereditary X-linked juvenile retinoschisis: A review of the role of Müller cells. Arch Ophthalmol 120: 979-984, 2002.

4. Molday RS, Kellner U and Weber BH: X-linked juvenile retinoschisis: Clinical diagnosis, genetic analysis, and molecular mechanisms. Prog Retin Eye Res 31: 195-212, 2012.

5. Tantri A, Vrabec TR, Cu-Unjieng A, Frost A, Annesley WH Jr and Donoso LA: X-linked retinoschisis: A clinical and molecular genetic review. Surv Ophthalmol 49: 214-230, 2004.

6. Sikkink SK, Biswas S, Parry NRA, Stanga PE and Trump D: X-linked retinoschisis: An update. J Med Genet 44: 225-232, 2007

7. Renner AB, Kellner U, Fiebig B, Cropp E, Foerster MH and Weber BH: ERG variability in X-linked congenital retinoschisis patients with mutations in the RS1 gene and the diagnostic importance of fundus autofluorescence and OCT. Doc Ophthalmol 116: 97-109, 2008.

8. Yu J, Ni Y, Keane PA, Jiang C, Wang W and Xu G: Foveomacular schisis in juvenile X-linked retinoschisis: An optical coherence tomography study. Am J Ophthalmol 149: 973-978.e2, 2010.

9. Wang T, Waters CT, Rothman AMK, Jakins TJ, Römisch K and Trump D: Intracellular retention of mutant retinoschisin is the pathological mechanism underlying X-linked retinoschisis. Hum Mol Genet 11: 3097-3105, 2002.

10. Wu WW, Wong JP, Kast J and Molday RS: RS1, a discoidin domain-containing retinal cell adhesion protein associated with $\mathrm{X}$-linked retinoschisis, exists as a novel disulfide-linked octamer. J Biol Chem 280: 10721-10730, 2005

11. Molday LL, Hicks D, Sauer CG, Weber BHF and Molday RS: Expression of X-linked retinoschisis protein RS1 in photoreceptor and bipolar cells. Invest Ophthalmol Vis Sci 42: 816-825, 2001.

12. Tolun G, Vijayasarathy C, Huang R, Zeng Y, Li Y, Steven AC, Sieving PA and Heymann JB: Paired octamer rings of retinoschisin suggest a junctional model for cell-cell adhesion in the retina. Proc Natl Acad Sci USA 113: 5287-5292, 2016.

13. Yzer S, Barbazetto I, Allikmets R, van Schooneveld MJ Bergen A, Tsang SH, Jacobson SG and Yannuzzi LA: Expanded clinical spectrum of enhanced S-cone syndrome. JAMA Ophthalmol 131: 1324-1330, 2013.

14. Sohn EH, Chen FK, Rubin GS, Moore AT, Webster AR and MacLaren RE: Macular function assessed by microperimetry in patients with enhanced S-cone syndrome. Ophthalmology 117: 1199-1206.e1, 2010

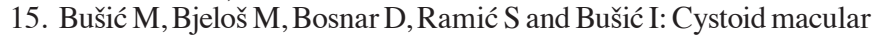
lesions are resistant to topical dorzolamide treatment in enhanced S-cone syndrome child. Doc Ophthalmol 132: 67-73, 2016.

16. Salvatore S, Fishman GA and Genead MA: Treatment of cystic macular lesions in hereditary retinal dystrophies. Surv Ophthalmol 58: 560-584, 2013.

17. Sigler EJ: Microcysts in the inner nuclear layer, a nonspecific SD-OCT sign of cystoid macular edema. Invest Ophthalmol Vis Sci 55: 3282-3284, 2014.
18. Murata N, Togano T, Miyamoto D, Ochiai S and Fukuchi T: Clinical evaluation of microcystic macular edema in patients with glaucoma. Eye (Lond) 30: 1502-1508, 2016.

19. Gregori NZ, Berrocal AM, Gregori G, Murray TG, Knighton RW, Flynn HW Jr, Dubovy S, Puliafito CA and Rosenfeld PJ: Macular spectral-domain optical coherence tomography in patients with X linked retinoschisis. Br J Ophthalmol 93: 373-378, 2009.

20. Prenner JL, Capone A Jr, Ciaccia S, Takada Y, Sieving PA and Trese MT: Congenital X-linked retinoschisis classification system. Retina 26: S61-S64, 2006.

21. Gregori NZ, Lam BL, Gregori G, Ranganathan S, Stone EM, Morante A, Abukhalil F and Aroucha PR: Wide-field spectraldomain optical coherence tomography in patients and carriers of X-linked retinoschisis. Ophthalmology 120: 169-174, 2013.

22. Atchaneeyasakul LO, Trinavarat A, Pituksung A, Jinda W, Thongnoppakhun W and Limwongse C: Mutations in the XLRS1 gene in Thai families with X-linked juvenile retinoschisis. Jpn J Ophthalmol 54: 89-93, 2010.

23. Li X, Ma X and Tao Y: Clinical features of X linked juvenile retinoschisis in Chinese families associated with novel mutations in the RS1 gene. Mol Vis 13: 804-812, 2007.

24. Kim SY, Ko HS, Yu YS, Hwang JM, Lee JJ, Kim SY, Kim JY, Seong MW, Park KH and Park SS: Molecular genetic characteristics of X-linked retinoschisis in Koreans. Mol Vis 15: 833-843, 2009.

25. Lesch B, Szabó V, Kánya M, Somfai GM, Vámos R, Varsányi B, Pámer Z, Knézy K, Salacz G, Janáky M, et al: Clinical and genetic findings in Hungarian patients with X-linked juvenile retinoschisis. Mol Vis 14: 2321-2332, 2008.

26. Riveiro-Alvarez R, Trujillo-Tiebas MJ, Gimenez-Pardo A, Garcia-Hoyos M, Lopez-Martinez MA, Aguirre-Lamban J, Garcia-Sandoval B, Vazquez-Fernandez del Pozo S, Cantalapiedra D, Avila-Fernandez A, et al: Correlation of genetic and clinical findings in Spanish patients with X-linked juvenile retinoschisis. Invest Ophthalmol Vis Sci 50: 4342-4350, 2009.

27. Eksandh LC, Ponjavic V, Ayyagari R, Bingham EL, Hiriyanna KT, Andréasson S, Ehinger B and Sieving PA: Phenotypic expression of juvenile X-linked retinoschisis in Swedish families with different mutations in the XLRS1 gene. Arch Ophthalmol 118: 1098-1104, 2000

28. Iannaccone A, Mura M, Dyka FM, Ciccarelli ML, Yashar BM, Ayyagari R, Jablonski MM and Molday RS: An unusual X-linked retinoschisis phenotype and biochemical characterization of the W112C RS1 mutation. Vision Res 46: 3845-3852, 2006.

29. Verbakel SK, van de Ven JP, Le Blanc LMP, Groenewoud JMM, de Jong EK, Klevering BJ and Hoyng CB: Carbonic anhydrase inhibitors for the treatment of cystic macular lesions in children with X-linked juvenile retinoschisis. Invest Ophthalmol Vis Sci 57: 5143-5147, 2016.

30. Marangoni D, Bush RA, Zeng Y, Wei LL, Ziccardi L, Vijayasarathy C, Bartoe JT, Palyada K, Santos M, Hiriyanna S, et al: Ocular and systemic safety of a recombinant AAV8 vector for X-linked retinoschisis gene therapy: GLP studies in rabbits and Rs1-KO mice. Mol Ther Methods Clin Dev 5: 16011, 2016.

31. Zeng Y, Petralia RS, Vijayasarathy C, Wu Z, Hiriyanna S, Song H, Wang YX, Sieving PA and Bush RA: Retinal structure and gene therapy outcome in retinoschisin-deficient mice assessed by spectral-domain optical coherence tomography. Invest Ophthalmol Vis Sci 57: OCT277-OCT287, 2016.

32. Bashar AE, Metcalfe AL, Viringipurampeer IA, Yanai A, Gregory-Evans CY and Gregory-Evans K: An ex vivo gene therapy approach in X-linked retinoschisis. Mol Vis 22: 718-733, 2016.

33. Apaolaza PS, Del Pozo-Rodríguez A, Solinís MA, Rodríguez JM, Friedrich U, Torrecilla J, Weber BH and Rodríguez-Gascón A: Structural recovery of the retina in a retinoschisin-deficient mouse after gene replacement therapy by solid lipid nanoparticles. Biomaterials 90: 40-49, 2016.

34. Study of RS1 ocular gene transfer for X-linked retinoschisis. www.clinicaltrials.gov. Last access December 2016.

35. Safety and efficacy of rAAV-hRS1 in patients with X-linked retinoschisis (XLRS). www.clinicaltrials.gov. Accessed December, 2016. 